\title{
Highly efficient hybrid warm white organic light-emitting diodes using a blue thermally activated delayed fluorescence emitter: exploiting the external heavy-atom effect
}

\author{
Dongdong Zhang, Lian Duan, Yunge Zhang, Minghan Cai, Deqiang Zhang and Yong Qiu
}

To attain high efficiencies in hybrid white organic light-emitting diodes (WOLEDs), mutual quenching of the fluorophors and phosphors should be minimized. Efforts have been devoted to reducing the triplet quenching of phosphors; however, the quenching of fluorophors by the external heavy-atom effect (EHA) introduced by the phosphors is often ignored. Here, we observed that conventional fluorophors and fluorophors with thermally activated delayed fluorescence (TADF) behave differently in the presence of EHA perturbers. The efficiencies of the conventional fluorophors suffer greatly from the EHA, whereas the TADF fluorophors exhibit negligible changes, which makes TADF materials ideal fluorophors for hybrid devices. WOLEDs using a blue TADF fluorophor and an orange phosphor achieve a maximum forward viewing external quantum efficiency of $19.6 \%$ and a maximum forward viewing power efficiency of $50.2 \mathrm{Im} \mathrm{W}^{-1}$, among the best values for hybrid WOLEDs. This report is the first time that the EHA effect has been considered in hybrid WOLEDs and that a general strategy toward highly efficient hybrid WOLEDs with simple structures is proposed.

Light: Science \& Applications (2015) 4, e232; doi:10.1038/Isa.2015.5; published online 2 January 2015

Keywords: external heavy-atom effect; hybrid white OLEDs; thermally activated delayed fluorescence

\section{INTRODUCTION}

Recent conceptual advancements have led to many exciting approaches to white organic light-emitting diodes (WOLEDs). ${ }^{1-4}$ For practical use, high efficiency and long lifetime are the most critical parameters. Blue phosphors can obtain high luminous efficiency but suffer from short lifetime, while blue fluorophors are exactly the opposite. ${ }^{5,6}$ Efforts have been devoted to resolve the tradeoff between the high efficiency and long lifetime by combining the advantages of phosphors and fluorophors, that is, by fabricating hybrid WOLEDs with blue fluorophors and green/red or orange phosphors. ${ }^{6-9}$

To attain high efficiencies in hybrid WOLEDs, mutual quenching of the fluorophors and phosphors should be minimized. It has been long realized that the quenching of the green and red phosphorescence by the low triplet energy fluorophors is one of the main losses of excitation energy in these devices. ${ }^{6}$ Therefore, a 'triplet harvesting' strategy by doping a blue fluorophor and red/green phosphors in separated regions of high-triplet-energy host materials has been proposed ${ }^{1,10}$ and is further improved by incorporating blue fluorophors with high triplet energy $\left(\mathrm{T}_{1}\right)$ to prevent the undesirable 'back energy transfer' from the phosphors to the blue fluorophors. ${ }^{2,7,8}$

However, the heavy metal in the phosphors introduce an external heavy-atom effect (EHA) in the emissive layer (EML) and may lead to quenching of the fluorescence due to the increased singlet $\left(\mathrm{S}_{1}\right)$-triplet $\left(\mathrm{T}_{1}\right)$ intersystem crossing (ISC) rate $\left(k_{\mathrm{ISC}}\right)$ constant and the radiative or non-radiative decay rate of triplet excitons $\left(k_{\mathrm{p}}\right)$ of the fluorophors. ${ }^{11-13}$ This process could be another possible mechanism of energy loss in hybrid WOLEDs, especially for the WOLED with a single EML, even though this process has been ignored in previous studies. ${ }^{1,2,14}$ The quenching process between the fluorophor and the EHA perturber is rather complicated and not fully understood. ${ }^{12}$ Multiple factors would affect the fluorescence quenching, such as the temperature, the distance between the fluorophor and the EHA perturber, and the nature of the fluorophor and perturber, respectively. ${ }^{12,15}$ Berberan-Santos et al. reported that even though the intrinsic quenching constants are very similar for phenanthrene-iodide and C70-bromobenzene systems, for phenanthrene-iodide, the quenching effect is high, while for $\mathrm{C} 70$-bromobenzene, the quenching effect is low as the competing decay processes are fast. ${ }^{15}$ The same group of authors also observed that both $k_{\mathrm{ISC}}$ and the rate of reverse ISC $\left(k_{\mathrm{RISC}}\right)$ of C70 are significantly improved when an EHA perturber is introduced, as the singlet-triplet split $\left(\Delta E_{\mathrm{ST}}\right)$ of $\mathrm{C} 70$ is very small. ${ }^{11}$ Notably, the ratio of the thermally activated delayed fluorescence (TADF) to the prompt fluorescence is higher than the unperturbed C70 at elevated temperatures, ${ }^{11}$ suggesting that the loss in TADF is less 
significant than that in the prompt fluorescence, most likely due to the existence of the competing $\mathrm{T}_{1} \rightarrow \mathrm{S}_{1} \rightarrow \mathrm{S}_{0}$ channel even though the radiative decay rate of $C 70 S_{1}$ is low. ${ }^{16}$ Therefore, a conventional fluorophor with a large $\Delta E_{\mathrm{ST}}$ and a TADF fluorophor would behave differently in the presence of an EHA perturber, as shown in Supplementary Fig. S1a and S1b. It is conceivable that recovery of the total photoluminescence (PL) efficiency in the presence of an EHA perturber might be possible for a fluorophor with both a small $\Delta E_{\mathrm{ST}}$ and a high radiative decay rate of $S_{1}$.

Only recently, Adachi et al. developed efficient pure organic TADF materials with small $\Delta E_{\mathrm{ST}}$, while maintaining a high radiative decay rate $\left(k_{\mathrm{r}}\right)$ by carefully tuning the overlap of the highest occupied molecular orbital and the lowest unoccupied molecular orbital. ${ }^{17-19}$ Therefore, it would be important to understand how a TADF fluorophor would perform in the presence of a phosphorescent EHA perturber.

Here, the effect of phosphorescent EHA perturbers on the PL behaviors of a TADF fluorophor is studied for the first time. It is observed that the TADF fluorophor is much less sensitive to the EHA perturber than the conventional fluorophors due to the almost unchanged $k_{\text {ISC }}$ and enhanced $k_{\mathrm{RISC}}$. By doping a yellow phosphor and a blue fluorophor with TADF into a single EML, hybrid WOLEDs with a maximum forward-viewing external quantum efficiency $\left(\mathrm{EQE}_{\max }\right)$ of $19.6 \%$ and a maximum forward-viewing power efficiency $\left(\mathrm{PE}_{\max }\right)$ of $50.2 \mathrm{~lm} \mathrm{~W} \mathrm{~W}^{-1}$ are achieved, The total $\mathrm{EQE}_{\max }$ of $33.3 \%$ and total $\mathrm{PE}_{\max }$ of $85.3 \mathrm{~lm} \mathrm{~W} \mathrm{~W}^{-1}$ were also recorded using an integrating sphere. The high efficiency is achieved by minimizing the possible quenching processes between the phosphor and fluorophor.

\section{MATERIALS AND METHODS}

The TADF fluorophor, 1,4-dicyano-2,3,5,6-tetrakis(3,6-diphenylcarbazol-9yl)benzene (t-Bu4CzTPN), was synthesized according to the method reported in the literature, ${ }^{19}$ with 3,6-diphenylcarbazol being replaced by 3,6-di-tert-butylcarbazole. Dipyrazino[2,3-f:2',3'-h]quinoxaline-2,3,6,7,10,11-hexacarbonitrile (HATCN) was purchased from LG Chem China Investment Co., Ltd. (Beijing, China), while $N, N^{\prime}$-bis(1-naphthalenyl)$N, N^{\prime}$-diphenyl-[1,1'-biphenyl]-4,4'-diamine (NPB) was purchased from Beijing Visionox Technology Co. Ltd. (Beijing, China). N,N,N-tris(4-(9carbazolyl)phenyl)amine (TCTA), 1,3-bis(9H-carbazol-9-yl)benzene (mCP) and 3-(1,1'-biphenyl-4-yl)-5-(4-tert-butylphenyl)-4-phenyl-4H-1,2,4-triazole (TAZ) were purchased from Jilin Optical and Electronic Materials Co. Ltd. (Changchun, Jilin province, China). The $2 \mathrm{CzPN}$ was purchased from Xi' an Polymer Light Technology Co. Ltd. (Xi'an, Shanxi province, China).

\section{PL characterization}

Ultraviolet-visible absorption spectra were recorded using an Agilent 8453 spectrophotometer. The films were spin coated on the quartz substrate using dichloromethane as the solvent, which was volatilized before the measurement. The concentration of $\mathrm{mCP}$ was $10 \mathrm{mg} \mathrm{mL}^{-1}$. The thicknesses of the films have little effect on their PL characters. The PL efficiencies and spectra of the films were measured using an absolute photoluminescence quantum yield measurement system (Hamamatsu C11347). The PL transient decay curves of the films were measured using a transient spectrometer (Edinburg FL920P).

\section{Device characterization}

The device structure of the hybrid WOLEDs was ITO/HATCN $(5 \mathrm{~nm}) /$ NPB $(40 \mathrm{~nm}) / \mathrm{TCTA}(10 \mathrm{~nm}) / \mathrm{mCP}(11 \mathrm{~nm}): 6 \mathrm{wt}-\%, 10 \mathrm{wt} \%$ and 20 wt- $\% \quad 2 \mathrm{CzPN}: \quad 0.2$ wt- $\%$ PO-01/TAZ $\quad(40 \mathrm{~nm}) / \mathrm{LiF} \quad(0.5 \mathrm{~nm}) / \mathrm{Al}$ $(150 \mathrm{~nm})$. The OLEDs were fabricated by thermal evaporation under high vacuum $\left(\sim 7 \times 10^{-4} \mathrm{~Pa}\right)$ onto clean ITO-coated glass substrates. The forward-viewing electrical characteristics of the devices were measured using a Keithley 2400 source meter, while the total ones were measured using an integrating sphere (Bluefly illumia 610). The electroluminescence spectra and luminance of the devices were obtained on a PR650 spectrometer. All the device fabrication and characterization steps were performed at room temperature under ambient laboratory conditions. For measurement of the transient electroluminescence characteristics, short-pulse excitation with a pulse width of $15 \mu$ s was generated using an Agilent $8114 \mathrm{~A}$ pulse generator. The amplitude of the pulse was $10 \mathrm{~V}$, and the baseline was $-3 \mathrm{~V}$. The period was $50 \mu \mathrm{s}$, the delayed time was $25 \mu \mathrm{s}$ and the duty cycle was $30 \%$. The decay curves of the devices were detected using an Edinburg FL920P transient spectrometer.

\section{RESULTS AND DISCUSSION}

To compare the effect of EHA on the TADF materials and conventional fluorophors, a TADF fluorophor ( $\mathrm{t}-\mathrm{Bu} 4 \mathrm{CzTPN})$ and two conventional fluorophors, bis(10-hydroxybenzo[h] quinolinato)beryllium complex $\left(\mathrm{Bebq}_{2}\right)$ and tris(8-hydroxyquinolinato)aluminum ( $\left.\mathrm{Alq}_{3}\right)$, were selected. Here, $\mathrm{Alq}_{3}$ was selected as a widely used conventional fluorophor, while $\mathrm{Bebq}_{2}$ was selected because it possesses high triplet energy with a small $\Delta E_{\mathrm{ST}}$ of $0.2 \mathrm{eV},{ }^{20}$ but not sufficient for TADF emission. The emission of the latter was approximately $490 \mathrm{~nm}$, which can represent the high triplet blue fluorophors used in hybrid WOLEDs. Dichloro(1,5-cyclooctadiene)platinum(II) $\left(\mathrm{Pt}(\mathrm{COD}) \mathrm{Cl}_{2}\right)$, iridium(III) [bis(4,6-difluorophenyl)-pyridinato-N, $\mathrm{C}^{2^{\prime}}$ ]picolinate (Firpic) and tri$\mathrm{s}$ (phenylpyrazole)iridium $\left(\operatorname{Ir}(\mathrm{ppz})_{3}\right)$ were used as EHA perturbers for the fluorophors. The molecule structures are shown in Figure 1a. To solely determine the effect of the EHA on the PL characteristics of the fluorophors, the excitation wavelength should be carefully selected to ensure that in the experiment, the phosphors cannot be excited and act only as EHA perturbers. Therefore, excitation wavelengths of $490 \mathrm{~nm}$, $430 \mathrm{~nm}$ and $430 \mathrm{~nm}$ were selected for Firpic, $\operatorname{Ir}(\mathrm{ppz})_{3}$ and $\operatorname{Pt}(\mathrm{COD}) \mathrm{Cl}_{2}$, respectively, as observed in Figure $1 \mathrm{~b}$ and 1c. To study the EHA in a rigid medium, ${ }^{13} \mathrm{mCP}$ was used as a host here. The absorption spectrum of pure mCP shown in Supplementary Fig. S2 indicates that the $\mathrm{mCP}$ cannot be excited with an excitation wavelength of $430 \mathrm{~nm}$ or $490 \mathrm{~nm}$, which rules out the interaction between the $\mathrm{mCP}$ and EHA perturbers. The concentration of the fluorophors is fixed at $5 \mathrm{wt}-\%$, while those of the phosphors are steadily increasing.

The efficiencies of the films of mCP: $5 \mathrm{wt}-\%$ fluorophors: $x \mathrm{wt}-\%$ ( 0 ; $5 ; 10 ; 15 ; 20 ; 25)$ phosphors are measured. As observed in Figure 2a and Supplementary Fig. S3, compared with the sharply reduced efficiencies of $\mathrm{Bebq}_{2}$ or $\mathrm{Alq}_{3}$, with increasing concentration of the phosphor, the PL efficiencies of t-BuCzTPN are only slightly affected. The Stern-Volmer plots for quenching of these fluorophors by phosphors are presented in Figure $2 \mathrm{~b}$ and Supplementary Fig. S3. The plots clearly demonstrate that the efficiency of $\mathrm{Bebq}_{2}$ and $\mathrm{Alq}_{3}$ are significantly quenched, whereas that of t-Bu4CzTPN is only slightly affected. All the spectra of these films are presented in Supplementary Fig. S4. The spectra of these fluorophors are identical as the concentration of the EHA perturbers increases, indicating that the effect of the polarity changing can be ruled out, ${ }^{18}$ and therefore, the change in the PL characteristics of these films is mainly due to the EHA effect.

For conventional fluorescence processes, the triplet excitons formed through ISC cannot be further recycled, and thus, the fluorescence efficiency $\left(\phi_{\mathrm{F}}\right)$ can be expressed as ${ }^{21}$

$$
\phi_{\mathrm{F}}=\frac{k_{\mathrm{r}}}{k_{\mathrm{r}}+k_{\mathrm{ISC}}+k_{\mathrm{nr}}}
$$

Here, when the EHA perturber is introduced, $k_{\mathrm{ISC}}$ is greatly increased, while $k_{\mathrm{r}}$ and the nonradiative rate of singlet $\left(k_{\mathrm{nr}}\right)$ are not 
a<smiles>ClP1(Cl)(Cl)C2CCCC1C2</smiles>

$\mathrm{Pt}(\mathrm{COD}) \mathrm{Cl}_{2}$

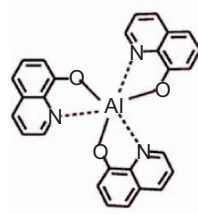

$\mathrm{Alq}_{3}$

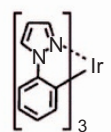

$\operatorname{Ir}(\mathrm{ppz})_{3}$

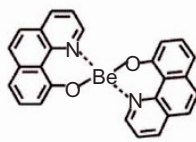

$\mathrm{Bebq}_{2}$

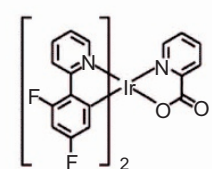

Firpic

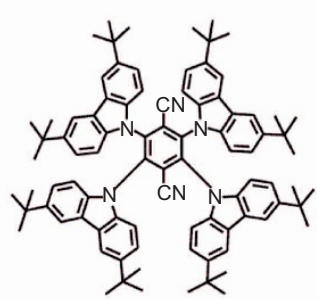

t-Bu4CzTPN
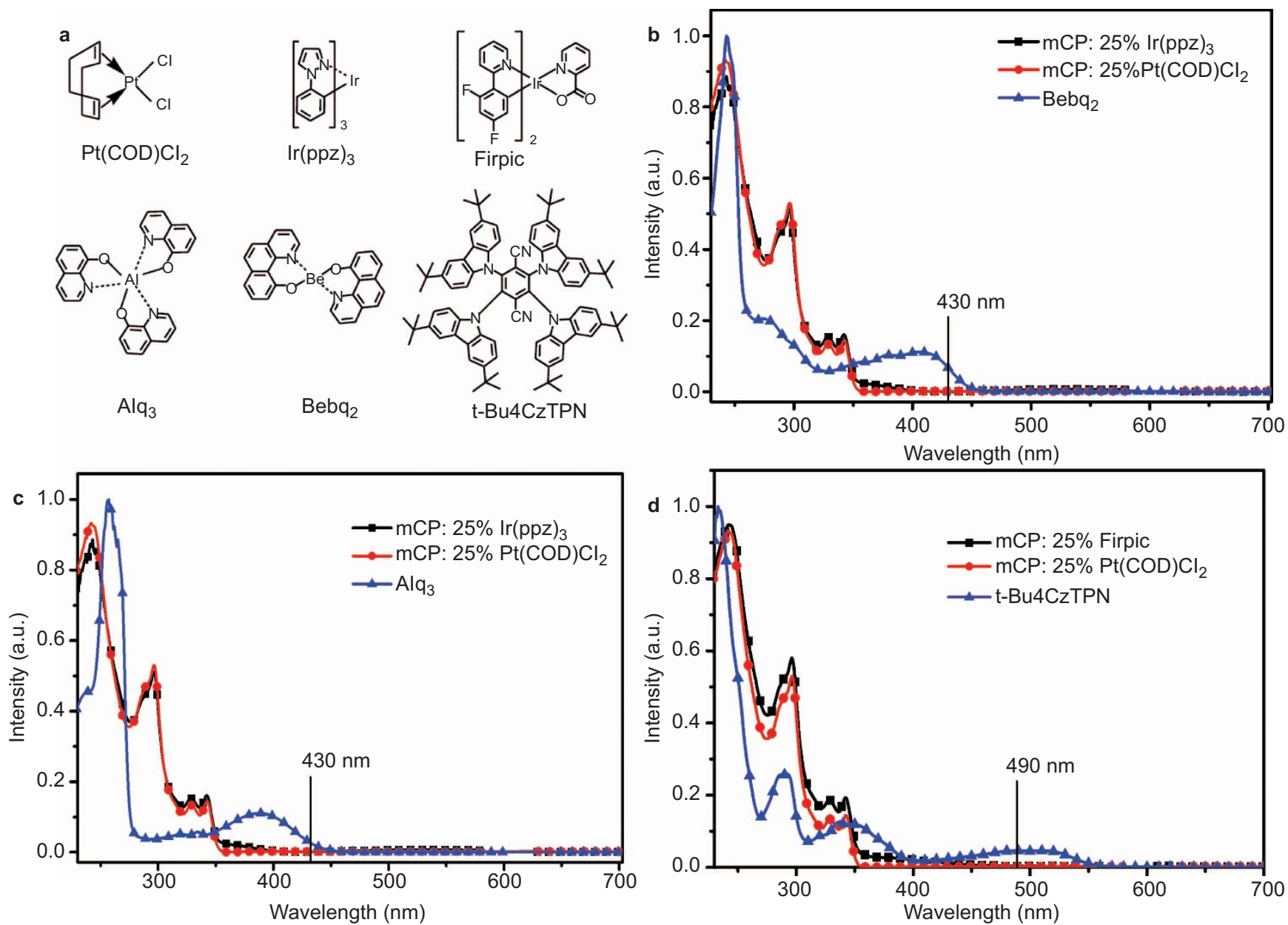

Figure 1 (a) The absorption spectra of $\operatorname{Ir}(\mathrm{ppz})_{3}, \mathrm{Pt}(\mathrm{COD}) \mathrm{Cl}_{2}$ in $\mathrm{mCP}$ : perturber films and $\mathrm{Bebq}_{2}$ in dichloromethane solution. (b) The absorption spectra of Ir(ppz) $)_{3}$, $\mathrm{Pt}(\mathrm{COD}) \mathrm{Cl} \mathrm{C}_{2}$ in mCP: perturber films and $\mathrm{Alq}_{3}$ in dichloromethane solution. (c) The absorption spectra of Firpic, $\mathrm{Pt}(\mathrm{COD}) \mathrm{Cl}_{2}$ in $\mathrm{mCP}$ : perturber films and t-Bu4CzTPN in dichloromethane solution.

affected, resulting in significantly reduced $\phi_{\mathrm{F}}$. Therefore, the sharply reduced efficiency of $\mathrm{Bebq}_{2}$ or $\mathrm{Alq}_{3}$ is due to the increased $k_{\mathrm{ISC}}$ with the introduction of the EHA perturber; for a stronger EHA, more excitons are lost, resulting in significant efficiency quenching. As observed in Figure $2 \mathrm{c}$ and Supplementary Fig. S5c, with the increasing concentration of $\operatorname{Ir}(\mathrm{ppz})_{3}$, the lifetime of the decay curves of $\mathrm{Bebq}_{2}$ and $\mathrm{Alq}_{3}$ are gradually decreased. The same trend was also observed when $\mathrm{Pt}(\mathrm{COD}) \mathrm{Cl}_{2}$ was used as the EHA perturber (Supplementary Fig. S5). The lifetime of the singlet excitons is expressed by ${ }^{22}$

$$
\tau=\frac{1}{k_{\mathrm{r}}+k_{\mathrm{ISC}}+k_{\mathrm{nr}}}
$$

Because $k_{\mathrm{r}}$ and $k_{\mathrm{nr}}$ are not affected, $k_{\mathrm{ISC}}$ should be greatly increased, resulting in a reduced lifetime of the singlet excitons and confirming our hypothesis above. The increase of $k_{\text {ISC }}$ can be calculated using Equation (2) and is shown in Supplementary Table S1. More triplet excitons will be formed in these fluorophors, and the radiative decay of the triplet excitons will be improved; therefore, such a system has been widely used to measure the triplet energies of fluorophors. ${ }^{23}$ However, as observed in Equation (1), if $k_{\mathrm{r}}$ of the fluorophors is much higher than the EHA-enlarged $k_{\mathrm{ISC}}$, the effect of EHA on $\phi_{\mathrm{F}}$ will be insignificant.

For the TADF fluorophors, as observed in Figure $2 \mathrm{~d}$ and Supplementary Fig. S5, the lifetimes of the prompt decay curves of t-Bu4CzTPN are almost the same with or without EHA perturbers
(17 ns for mCP: t-Bu4CzTPN: Firpic, see Supplementary Table S1), indicating that $k_{\mathrm{ISC}}$ of the fluorophors is less sensitive to the EHA perturbers. The reason may be attributed to the high intrinsic $k_{\text {ISC }}$ of the TADF fluorophors without the perturber due to their small $\Delta E_{\mathrm{ST}}$. As reported, the EHA will only affect the pre-exponential factor but not the activation energy. ${ }^{12}$ The increase of $k_{\text {ISC }}$ when an EHA perturber is introduced is rather limited for the $S_{1} \leftrightarrow T_{1}$ transition compared with the intrinsically high $k_{\mathrm{ISC}}$ of the TADF fluorophors. To further demonstrate that $k_{\mathrm{ISC}}$ is insensitive to the EHA perturber, the transient decay curves of $\mathrm{t}-\mathrm{Bu} 4 \mathrm{CzTPN}$ in toluene solution without degassing as the concentration of Firpic increased were also measured (Supplementary Fig. S6). The lifetime of the transient decay curves remains the same (10 ns, see Supplementary Table S1) as the concentration of Firpic increases, further demonstrating that the $k_{\mathrm{ISC}}$ of the fluorophors is less sensitive to the EHA perturber. The increase of $k_{\mathrm{RISC}}$ as observed for C70 derivatives ${ }^{11}$ with the introduction of the EHA perturber, is the same as for $\mathrm{t}-\mathrm{Bu} 4 \mathrm{CzTPN}$ with a small $\Delta E_{\mathrm{ST}}$ because the EHA manifests itself mainly by an increase of the $\mathrm{S}_{\mathrm{n}} \rightarrow \mathrm{T}_{\mathrm{n}}$ or $\mathrm{S}_{\mathrm{n}} \leftarrow \mathrm{T}_{\mathrm{n}}$ ISC rate constants. The small reduction of the PL efficiency of t-Bu4CzTPN can be attributed to the increased nonradiative decay rate of the triplet $\left(k_{\mathrm{np}}\right)$ when the EHA perturber is introduced. ${ }^{12}$ In contrast, when $\mathrm{Pt}(\mathrm{COD}) \mathrm{Cl}_{2}$ is used, the $\mathrm{PL}$ efficiency of $\mathrm{t}-\mathrm{Bu} 4 \mathrm{CzTPN}$ is slightly increased (Figure $2 \mathrm{a}$ ) within a certain range of $\mathrm{Pt}(\mathrm{COD}) \mathrm{Cl}_{2}$ concentration. The different behaviors can be interpreted 

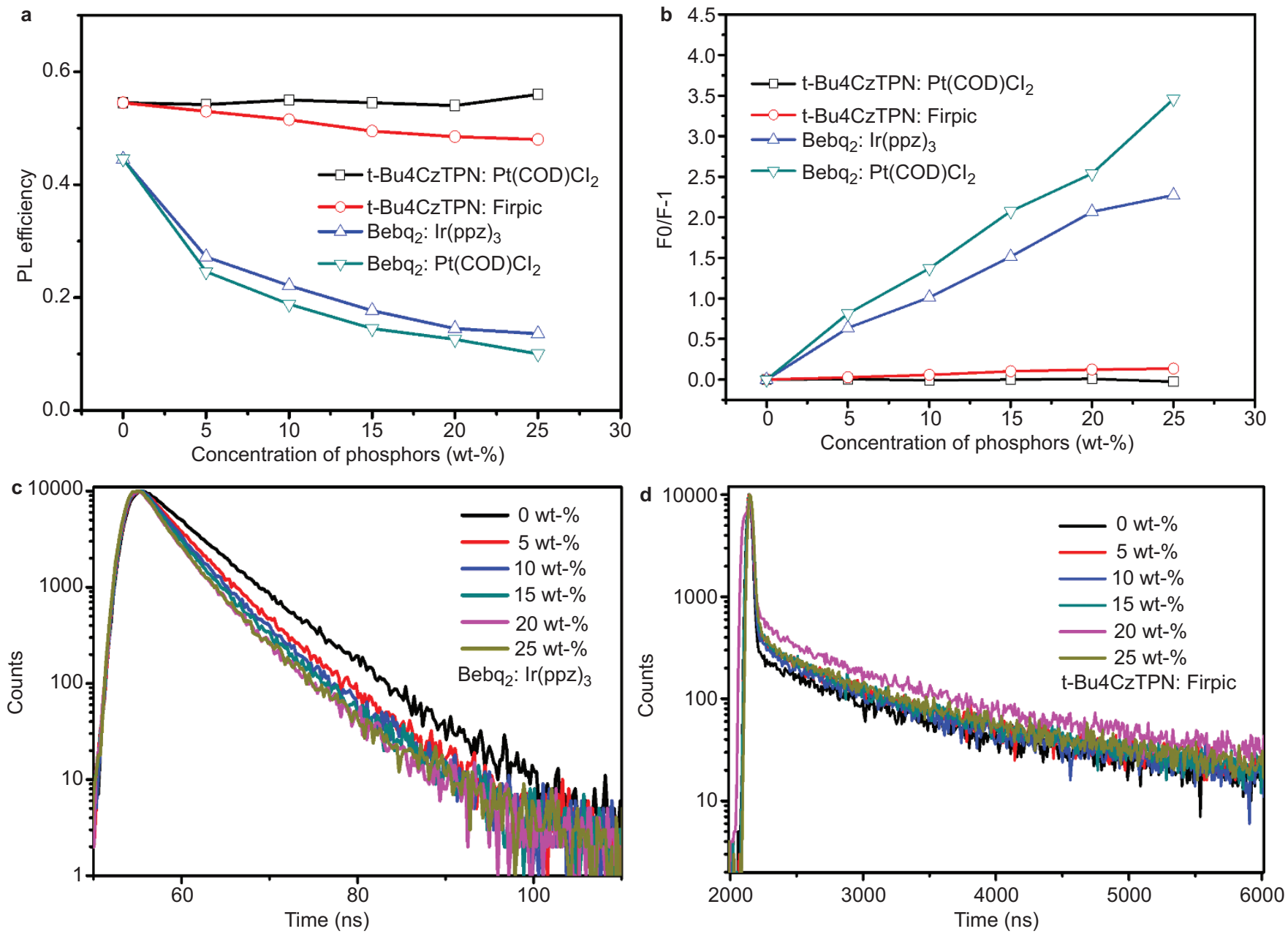

Figure 2 (a) The PL efficiencies of the films of mCP: fluorophors: EHA perturbers. (b) The Stern-Volmer plots for quenching of these fluorophors by EHA perturbers. FO stands for the PL efficiency of the fluorophors without EHA perturbers while $F$ is the PL efficiency with EHA perturbers. (c) The transient decay curves of films of mCP: $\mathrm{Bebq}_{2}: \operatorname{Ir}(\mathrm{ppz})_{3}$ measured at $490 \mathrm{~nm}$ with an excitation wavelength of $430 \mathrm{~nm}$. (d) The transient decay curves of films of mCP: t-Bu4CzTPN: Firpic measured at $576 \mathrm{~nm}$ with an excitation wavelength of $490 \mathrm{~nm}$. EHA, external heavy-atom effect; PL, photoluminescence.

as the combinational effects of the processes of TADF fluorophors affected by the EHA perturbers. This result demonstrates that under some circumstances, if the $k_{\mathrm{np}}$ is not increased significantly when the EHA perturber is added, the PL efficiency of the TADF fluorophors can even be improved. The combinational effects of ISC, RISC and nonradiative decay processes of the TADF fluorophors under the effect of EHA perturbers causes the PL efficiencies of these TADF fluorophors to be almost unchanged or even to have the potential to be promoted under certain circumstances, indicating that TADF fluorophors and phosphors may offer an ideal solution toward high efficiency and stable white OLEDs.

The importance of constructing hybrid WOLEDs is the selection of guest emitters and its host. Here, the TADF blue fluorophor, $2 \mathrm{CzPN}$, is selected due to its high PL efficiency and $\mathrm{EQE}_{\max }$ of $13.6 \%$ in the optimized blue device. ${ }^{24}$ A commonly used yellow phosphor, PO01 , is selected as the complementary phosphor. ${ }^{25}$ The efficiencies of TADF materials are sensitive to the triplets of the hosts; hence, $\mathrm{mCP}$ with high triplet energy is selected. ${ }^{24}$ Before the fabrication of devices, the PL efficiencies of films of mCP: $10 \mathrm{wt}-\%$ 2CzPN: $x$ wt- $\%$ PO-01 (where $x=0,0.2,0.4,0.6,0.8,1.0$ ) were measured, as observed in Supplementary Fig. S7b. The efficiencies of mCP: $x$ wt-\% PO-01 films were also measured (Supplementary Fig. S7a). The ratio of the blue emission and orange emission can be calculated from the integral of their own spectra, and hence, the PL efficiency of the mCP: $2 \mathrm{CzPN}$ : PO-01 without EHA perturber can be calculated (Supplementary Fig. S7). As observed in Supplementary Table S2, the experimental PL efficiencies are higher than the theoretical ones calculated using the spectra of those films. The reason may be attributed to the increased PL efficiency of 2CzPN due to the EHA perturber of PO-01. The EHA effect may increase the $\phi_{\mathrm{RISC}}$ of $2 \mathrm{CzPN}$ as we discuss above. For comparison, the interactions between $\mathrm{Bebq}_{2}$ and $\mathrm{PO}-01$ are also measured because the emission of $\mathrm{Bebq}_{2}$ is close to $2 \mathrm{CzPN}$. As observed in Supplementary Fig. S8, the experimental PL efficiencies of $\mathrm{mCP}$ : 10 wt- $\%$ Bebq $_{2}: x$ wt- $\%$ PO-01 (where $x=0,0.2,0.4,0.6,0.8,1.0$ ) are lower than the calculated ones, indicating that the EHA effect may decrease the PL efficiency of $\mathrm{Bebq}_{2}$. Therefore, the positive synergy effects between $2 \mathrm{CzPN}$ and $\mathrm{PO}-01$ made them a promising combination to fabricate high-efficiency WOLEDs.

The structure of the device fabricated is shown in Figure 3a. Both the fluorophor and phosphor are codoped into $\mathrm{mCP}$ as the EML, which is sandwiched between the electron-transporting layer of the TAZ film and the electron/exciton-blocking layer of the TCTA film. Meanwhile, 

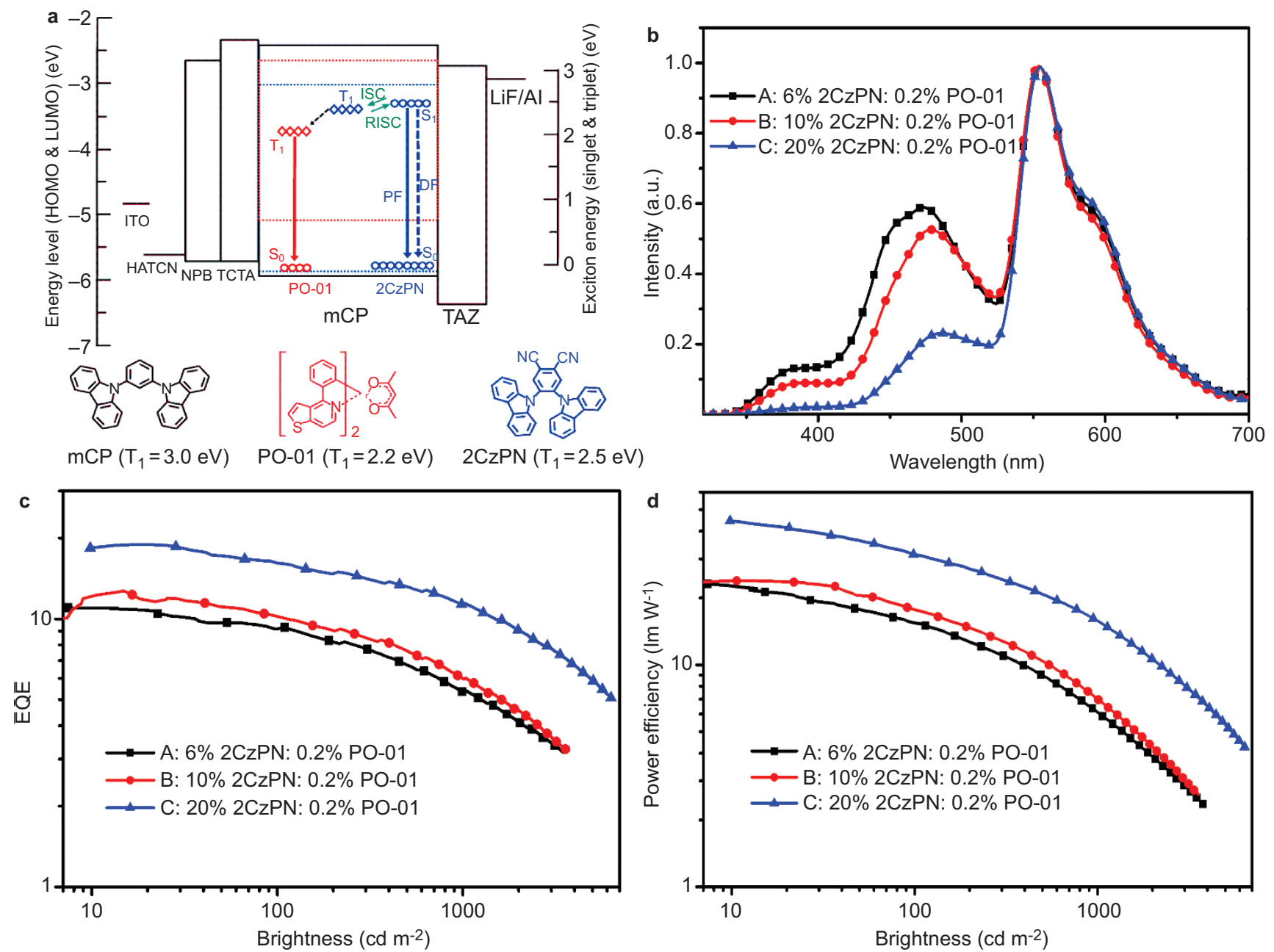

Figure 3 (a) The structure and energy diagram of the WOLEDs. (b) The spectra of the devices measured at 8 V. (c) EQE-brightness characteristics. (d) Power efficiencybrightness characteristics. EQE, external quantum efficiency; ISC, intersystem crossing; RISC, reverse intersystem crossing; WOLED, white organic light-emitting diodes.

NPB is introduced as the hole-transporting layer. Based on structural engineering to enhance the efficiency and spectral quality of the WOLEDs, optimization of the concentration of $2 \mathrm{CzPN}$ has been performed with that of PO-01 fixed at $0.2 \mathrm{wt}-\%$. The concentrations of $2 \mathrm{CzPN}$ are $6 \mathrm{wt}-\%, 10$ wt- $\%$ and 20 wt- $\%$ for device A, device B and device $\mathrm{C}$, respectively.

All the devices exhibit warm white emission with the spectra covering the entire range from $400 \mathrm{~nm}$ to $700 \mathrm{~nm}$, as observed in Figure $3 \mathrm{~b}$. With an increasing concentration of $2 \mathrm{CzPN}$, the intensity of blue emission is gradually reduced due to the more efficient energy transfer from $2 \mathrm{CzPN}$ to $\mathrm{PO}-01$. Here, the emission of the $\mathrm{mCP}$ host remains visible, indicating that energy transfer from $\mathrm{mCP}$ to $2 \mathrm{CzPN}$ or $\mathrm{PO}-01$ is not complete. As observed in Supplementary Fig. S9c-9e, the blue emission intensities of the devices are gradually increased with increasing voltage, similar to the previous reported hybrid WOLEDs. ${ }^{8}$ The Commission International de L'Eclairage coordinates of the white devices changed from $(0.38,0.44)$ at $4 \mathrm{~V}$ to $(0.33,0.38)$ at $10 \mathrm{~V}$ for device $\mathrm{A},(0.38,0.46)$ at $4 \mathrm{~V}$ to $(0.32,0.39)$ at $10 \mathrm{~V}$ for device $\mathrm{B}$ and $(0.43,0.49)$ to $(0.38,0.45)$ for device $\mathrm{C}$. The CRIs range from 53 at $4 \mathrm{~V}$ to 62 at $10 \mathrm{~V}$ for device $\mathrm{A}, 53$ at $4 \mathrm{~V}$ to 62 at $10 \mathrm{~V}$ for device $\mathrm{B}$ and 53 at $4 \mathrm{~V}$ to 62 at $10 \mathrm{~V}$ for device C. For light applications, the most important reference light source is the warm white standard illumination A with Commission International de L'Eclairage coordinates of $(0.445$, $0.405)$, exhibiting higher intensity in the orange region. ${ }^{6}$ In addition, candle-light-style OLEDs emitting yellowish orange light have been demonstrated to be a physiologically friendly light at night. ${ }^{26,27}$ Therefore, devices A-C are physiologically friendly light due to their relatively low intensities of blue emission.

The highest maximum forward viewing EQEs are 19.6\%, 12.1\% and $10.3 \%$ for devices with $20 \%$ (device C), $10 \%$ (device B) and $6 \% 2 \mathrm{CzPN}$ (device A), respectively (Figure $3 \mathrm{c}$ ). The low efficiency with lower $2 \mathrm{CzPN}$ dopant concentration is partly due to the inefficient energy transfer. The device with $20 \% 2 \mathrm{CzPN}$ produces a maximum forward viewing power efficiency of $50.2 \mathrm{~lm} \mathrm{~W}^{-1}$ (Figure 3d). Because illumination sources are typically characterized by their total emitted power, our WOLED exhibits a total $\mathrm{EQE}_{\max }$ of $33.3 \%$ and a total $\mathrm{PE}_{\max }$ of $85.3 \mathrm{~lm} \mathrm{~W} \mathrm{~W}^{-1}$ (measured using an integrating sphere), which roll off to $22.7 \%$ and $34.0 \mathrm{~lm} \mathrm{~W} \mathrm{~W}^{-1}$ at a high brightness of $1000 \mathrm{~cd} \mathrm{~m}^{-2}$. To the best of our knowledge, these values are among the best results reported thus far for hybrid white devices (see Table 1) and are even comparable to the values of all phosphor white devices. ${ }^{4}$ More importantly, considering the PL efficiency of mCP: 20 wt-\% 2CzPN: 0.2 wt-\% PO-01 of 0.752 and assuming an optical outcoupling efficiency of approximately $20 \%$ $30 \%,{ }^{28}$ the $\mathrm{EQE}_{\max }$ of $19.6 \%$ approaches the theoretical maximum value 
Table 1 Device performances of some hybrid WOLEDs

\begin{tabular}{|c|c|c|c|c|c|}
\hline & $\mathrm{EQE}_{\text {max }}^{\mathrm{a}}$ & $\mathrm{EQE}^{\mathrm{b}}{ }_{\text {max }}$ & $P E^{a}{ }_{\max }\left(\operatorname{lm~} W^{-1}\right)$ & $P E^{\mathrm{b}}{ }_{\max }\left(\operatorname{Im~} \mathrm{W}^{-1}\right)$ & $\operatorname{CIE}(x, y)$ \\
\hline Ref 2 & - & $20.3 \%^{\mathrm{c}}$ & - & $57.6^{\mathrm{c}}$ & $(0.45,0.48)^{d}$ \\
\hline Ref 7 & - & $21.8 \%$ & - & 57.3 & $(0.48,0.44)^{d}$ \\
\hline Ref 8 & - & $26.6 \%$ & - & 67.2 & $(0.46,0.44)^{d}$ \\
\hline Device A & $10.3 \%$ & & 23.0 & & $(0.36,0.42)^{d}$ \\
\hline Device B & $12.1 \%$ & & 24.5 & & $(0.36,0.44)^{d}$ \\
\hline Device C & $19.6 \%$ & $33.3 \%$ & 50.2 & 85.3 & $(0.42,0.48)^{d}$ \\
\hline
\end{tabular}

Abbreviations: CIE, Commission International de L'Eclairage.

a Forward viewing efficiency.

b Total efficiency.

${ }^{c}$ Measured at $1000 \mathrm{~cd} \mathrm{~m}^{-2}$

${ }^{\mathrm{d}}$ Measured at $100 \mathrm{~cd} \mathrm{~m}^{-2}$.

$(15 \%-22 \%)$, which indicates that the number of triplet excitons wasted is negligible. For the blue TADF material, there are two parallel approaches to minimize the triplet excitons lost, as demonstrated in Figure 3a. On the one hand, because of the higher triplet energy of $2 \mathrm{CzPN}$ compared with that of PO-01, energy transfer from the triplet of $2 \mathrm{CzPN}$ to that of $\mathrm{PO}-01$ is energetically favorable, while the energy back transfer from $\mathrm{PO}-01$ is prohibited. On the other hand, as discussed above, due to its small $\Delta E_{\mathrm{ST}}$, the triplet excitons in $2 \mathrm{CzPN}$ can be thermally upconverted into the emissive singlet ones, hence further reducing triplet loss. For hybrid WOLEDs using conventional blue fluorophors with a large $\Delta E_{\mathrm{ST}}$, unity IQE can only be achieved when all the triplet excitons are used in phosphors, which results in the restriction for spectra tuning because the ratio of excitons used in blue fluorophors must be lower than $25 \%$. The method demonstrated here has the potential to allow free tuning of the emission spectra from cold white to warm white similar to the all phosphor devices, although only warm white emission is obtained for device A, and may achieve longer lifetimes than the all phosphors devices. ${ }^{29}$ The mutual quenching of the fluorophors and phosphors in such hybrid devices are minimized, making TADF materials adequate for use in high-efficiency hybrid WOLEDs.

Though the efficiency roll-off of device $\mathrm{C}$ is rather high with only $58 \%$ of the highest EQE remaining at the brightness of $1000 \mathrm{~cd} \mathrm{~m}^{-2}$, it remains less significant than the monochromatic device using $2 \mathrm{CzPN}$ as the emitter, as shown in Supplementary Fig. S10. This finding agrees well with former studies that singlet-triplet annihilation and triplet-triplet annihilation dominate efficiency roll-off for
$2 \mathrm{CzPN}$-based devices due to the long triplet lifetime of $2 \mathrm{CzPN}$ $(273 \mu \mathrm{s}) .^{30}$ The narrow recombination zone due to the use of a hole-dominant host of mCP may also account for the efficiency roll-off of the device. To demonstrate our idea, we fabricated another WOLED using a bipolar host of 2,6-bis(3-(9H-carbazol-9-yl)phenyl)pyridine (26DczPPy) with the same structure as device C. As observed in Supplementary Fig. S11, the efficiency roll-off is significantly reduced, with $85 \%$ of the highest efficiency remaining at a brightness of $1000 \mathrm{~cd} \mathrm{~m}^{-2}$, indicating that the performance of the hybrid WOLEDs can be improved by selecting adequate hosts.

To reveal the energy transfer process in the devices, the transient electroluminescence of devices $\mathrm{A}-\mathrm{C}$ was measured. For comparison, the transient decay curves of devices only doped with $2 \mathrm{CzPN}$ and PO01 were also measured. The transient decay curves for $2 \mathrm{CzPN}$ were measured at $480 \mathrm{~nm}$ for $2 \mathrm{CzPN}$ and at $560 \mathrm{~nm}$ for PO-01.

As observed in Figure $4 \mathrm{a}$, in the turn-off region, transient overshoots are observed from the decay curves at $480 \mathrm{~nm}$ after the voltage pulse was turned off, evidently due to charge trapping in the blue dopant. $^{31}$ Therefore, direct charge recombination on $2 \mathrm{CzPN}$ in the EML should occur. In addition, the recombination should also occur on the host because the emission of $\mathrm{mCP}$ is observed from the $\mathrm{EL}$ spectra, indicating that the energy of $2 \mathrm{CzPN}$ emission is also transferred from the host. Moreover, compared with blue devices using $2 \mathrm{CzPN}$ as the emitter, the ratio and lifetime of the delayed part of $2 \mathrm{CzPN}$ in the WOLEDs is increased rather than reduced. This result can be interpreted based on the EHA effect introduced by the
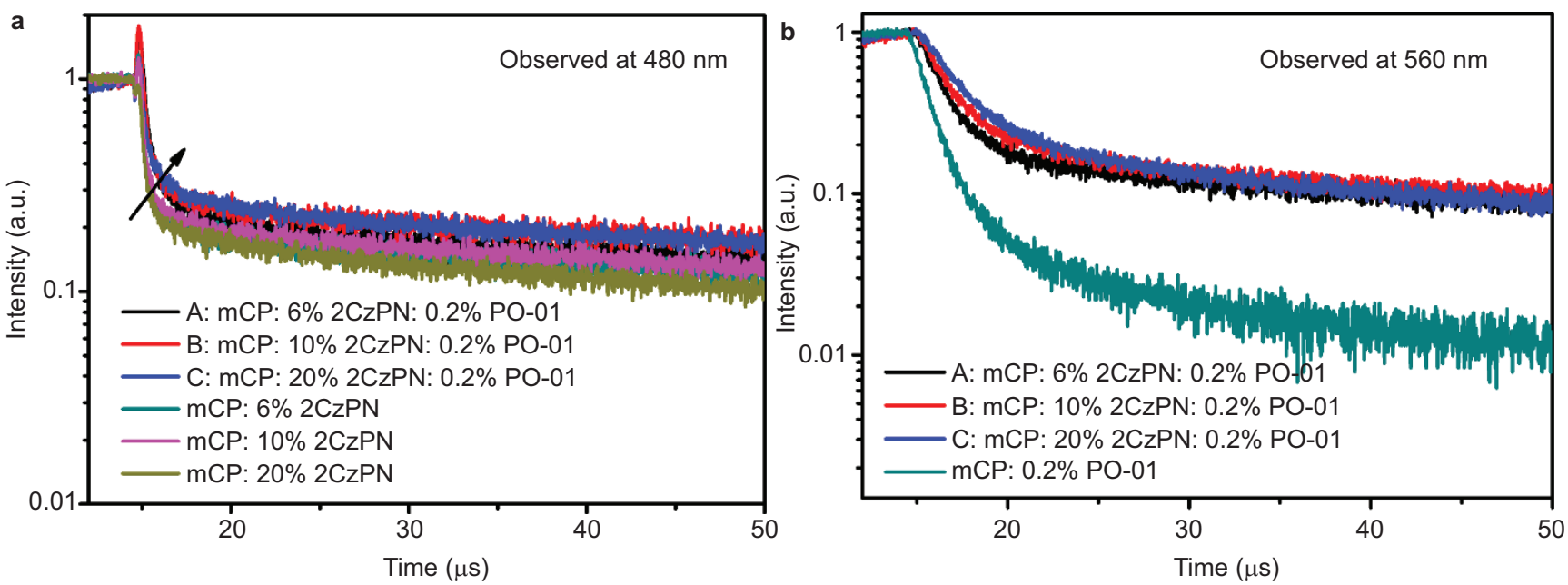

Figure 4 (a) The transient decay curves of devices observed at $480 \mathrm{~nm}$. (b) The transient decay curves of devices observed at $560 \mathrm{~nm}$. 
phosphors, as discussed above, which improves the random change between the excited singlets and triplets.

For PO-01, the excitons can be generated in three ways, that is, energy transfer from $\mathrm{mCP}$, energy transfer from $2 \mathrm{CzPN}$, or direct recombination of charges on PO-01. As observed in Figure 4b, no overshoots are observed in the decay curves of PO-01 in the WOLEDs, indicating that direct charge recombination on PO-01 is negligible. The energy transferred from $2 \mathrm{CzPN}$ to $\mathrm{PO}-01$ can be determined by the longer lifetime of PO-01 in WOLEDs compared with that of the device with only PO-01 as the emitter (Figure $4 \mathrm{~b}$ ). The long-lived triplet excitons of $2 \mathrm{CzPN}$ transferred to PO-01 results in the long lifetime of PO-01 emission. The energy transferred form mCP is also possible because excitons are also formed on the host, especially at higher driving voltages.

The operation lifetime of device $\mathrm{C}$ was measured at an initial brightness of $1000 \mathrm{~cd} \mathrm{~m}^{-2}$ under constant current. As observed in Supplementary Fig. S12a, the lifetime of device $\mathrm{C}$ is approximately $140 \mathrm{~min}$. The lifetimes of the monochromatic devices of $2 \mathrm{CzPN}$ or $\mathrm{PO}-01$ in the same device architecture are even shorter. Because PO-01 has been demonstrated to be a stable emitter, ${ }^{25}$ we believe that the instability of the $\mathrm{mCP}$ host should be one of the reasons for the short lifetime of the devices. Moreover, WOLED with 26DCzPPy as the host has a much longer lifetime than device $\mathrm{C}$ (Supplementary Fig. S12b), demonstrating that the stability of the hybrid OLEDs can be improved by selecting better host materials.

\section{CONCLUSIONS}

In conclusion, we have demonstrated that the PL efficiencies of the conventional fluorophors are effectively quenched by the EHA effect of the phosphors, while those of the TADF materials are less affected and may be slightly increased. Hybrid WOLEDs using a blue TADF fluorophor of $2 \mathrm{CzPN}$ and a yellow phosphor of PO-01 achieved an $\mathrm{EQE}_{\max }$ of $19.6 \%$ and a maximum $\mathrm{PE}_{\max }$ of $50.2 \mathrm{~lm} \mathrm{~W} \mathrm{~W}^{-1}$, corresponding to a total $\mathrm{EQE}_{\max }$ of $33.3 \%$ and a total $\mathrm{PE}_{\max }$ of $85.3 \mathrm{~lm} \mathrm{~W} \mathrm{~W}^{-1}$. Possible pathways of quenching, such as back energy transfer from the fluorophor to the phosphor and the quenching of the fluorescence of the fluorophor due to the presence of EHA, can be effectively blocked in this type of hybrid WOLEDs. This method opens a new avenue for achieving high-performance hybrid WOLEDs with simple structures.

\section{ACKNOWLEDGEMENTS}

We would like to thank the National Natural Science Foundation of China (Grant Nos. 51173096, 21161160447 and 61177023) for financial support.

1 Sun YR, Giebink NC, Kanno H, Ma BW, Thompson ME et al. Management of singlet and triplet excitons for efficient white organic light-emitting devices. Nature 2006; 440 : 908-912.

2 Schwartz G, Pfeiffer M, Reineke S, Walzer K, Leo K. Harvesting triplet excitons from fluorescent blue emitters in white organic light-emitting diodes. Adv Mater 2007; 19: 3672-3676.

3 Su SJ, Gonmori E, Sasabe H, Kido J. Highly efficient organic blue-and white-lightemitting devices having a carrier- and exciton-confining structure for reduced efficiency roll-off. Adv Mater 2008; 20: 4189-4194

4 Lee SY, Yasuda T, Yang YS, Zhang QS, Adachi C. Luminous butterflies: efficient exciton harvesting by benzophenone derivatives for full-color delayed fluorescence OLEDs. Angew Chem Int Ed 2014; 53: 6402-6406.

5 Baldo MA, O'Brien DF, You Y, Shoustikov A, Sibley S et al. Highly efficient phosphorescent emission from organic electroluminescent devices. Nature 1998; 395: 151-154.

6 Schwartz G, Reineke S, Rosenow TC, Walzer K, Leo K. Triplet harvesting in hybrid white organic light-emitting diodes. Adv Funct Mater 2009; 19: 1319-1333.
7 Zheng CJ, Wang J, Ye J, Lo MF, Liu XK et al. Novel efficient blue fluorophors with smal singlet-triplet splitting: hosts for highly efficient fluorescence and phosphorescence hybrid WOLEDs with simplified structure. Adv Mater 2013; 25: 2205-2211.

8 Ye J, Zheng CJ, Ou XM, Zhang XH, Fung MK et al. Management of singlet and triplet excitons in a single emission layer: a simple approach for a high-efficiency fluorescence/ phosphorescence hybrid white organic light-emitting device. Adv Mater 2012; 24: 3410-3414.

9 Sun N, Wang Q, Zhao YB, Chen YH, Yang DZ et al. High-performance hybrid white organic light-emitting devices without interlayer between fluorescent and phosphorescent emissive regions. Adv Mater 2014; 26: 1617-1621.

10 Schwartz G, Fehse K, Pfeiffer M, Walzer K, Leo K. Highly efficient white organic light emitting diodes comprising an interlayer to separate fluorescent and phosphorescent regions. Appl Phys Lett 2006; 89: 083509.

11 Baleizão C, Berberan-Santos MN. External heavy-atom effect on the prompt and delayed fluorescence of [70]fullerenes. Chem Phys Chem 2010; 11: 3133-3140.

12 Kearvell A, Wilkinson F. Fluorescence quenching and external spin-orbit coupling effects. Mol Cryst 1968; 4: 69-81.

13 Berberan-Santos MN. External heavy-atom effect on fluorescence kinetics. PhysChemComm 2000; 3: 18-23.

14 Baldo MA, Thompson ME, Forrest SR. High-efficiency fluorescent organic lightemitting devices using a phosphorescent sensitizer. Nature 2000; 403: 750-753.

15 Rae M, Fedorov A, Berberan-Santos MN. Fluorescence quenching with exponential distance dependence: application to the external heavy-atom effect. J Chem Phys 2003; 119: 2223-2231.

16 Berberan-Santos MN, Garcia JM. Unusually strong delayed fluorescence of $\mathrm{C}_{70}$. J AM Chem Soc 1996; 118: 9391-9394.

17 Sato K, Shizu K, Yoshimura K, Kawada A, Miyazaki H et al. Organic luminescent molecule with energetically equivalent singlet and triplet excited states for organic light-emitting diodes. Phys Rev Lett 2013; 110: 247401.

18 Ishimatsu R, Matsunami S, Shizu K, Adachi C, Nakano K et al. Solvent effect on thermally activated delayed fluorescence by 1,2,3,5-Tetrakis(carbazol-9-yl)-4,6dicyanobenzene. J Phys Chem A 2013; 117: 5607-5612.

19 Uoyama H, Goushi K, Shizu K, Nomura H, Adachi C. Highly efficient organic lightemitting diodes from delayed fluorescence. Nature 2012; 492: 234-240.

20 Jeon WS, Park TJ, Kim SY, Pode R, Jang J et al. Ideal host and guest system in phosphorescent OLEDs. Org Electron 2009; 10: 240-246.

21 Goushi K, Yoshida K, Sato K, Adachi C. Organic light-emitting diodes employing efficient reverse intersystem crossing for triplet-to-singlet state conversion. Nat Photonics 2012; 6: 253-258.

22 Zhang DD, Duan L, Li C, Li YL, Li HY et al. High-efficiency fluorescent organic lightemitting devices using sensitizing hosts with a small singlet-triplet exchange energy. Adv Mater 2014, 26: 5050-5055.

23 Tanaka I, Tabata Y, Tokito S. Observation of phosphorescence from tris(8hydroxyquinoline) aluminum thin films using triplet energy transfer from iridium complexes. Phys Rev B 2005; 71: 205207.

24 Masui K, Nakanotani H, Adachi C. Analysis of exciton annihilation in high-efficiency sky-blue organic light-emitting diodes with thermally activated delayed fluorescence. Org Electron 2013; 14: 2721-2726.

25 Zhang DD, Duan L, Li YL, Li HY, Bin ZY et al. Towards high efficiency and low roll-off orange electrophosphorescent devices by fine tuning singlet and triplet energies of bipolar hosts based on indolocarbazole/1, 3, 5-triazine hybrids. Adv Funct Mater 2014; 24: 3551-3561.

26 Jou JH, Hsieh CY, Tseng JR, Peng SH, Jou YC et al. Candle light-style organic lightemitting diodes. Adv Funct Mater 2013; 23: 2750-2757.

27 Stevens RG, Brainard GC, Blask DE, Lockley SW, Motta ME. Breast cancer and circadian disruption from electric lighting in the modern world. CA Cancer J Clin 2014; 64: 207218.

28 Smith LH, Wasey JA, Barnes WL. Light outcoupling efficiency of top-emitting organic light-emitting diodes. Appl Phys Lett 2004; 84: 2986-2988.

29 Nakanotani H, Masui K, Nishide J, Shibata T, Adachi C. Promising operational stability of high-efficiency organic light-emitting diodes based on thermally activated delayed fluorescence. Sci Rep 2013; 3: 2127.

30 Masui K, Nakanotani H, Adachi C. Analysis of exciton annihilation in high-efficiency sky-blue organic light-emitting diodes with thermally activated delayed fluorescence. Org Electron 2013; 14: 2721-2726.

31 Liu R, Gan ZQ, Shinar R, Shinar J. Transient electroluminescence spikes in small molecular organic light-emitting diodes, Phys Rev B 2011; 83: 245302.

\section{This work is licensed under a Creative Commons Attribution}

NonCommercial-NoDerivs 3.0 Unported License. The images or other third party material in this article are included in the article's Creative Commons license, unless indicated otherwise in the credit line; if the material is not included under the Creative Commons license, users will need to obtain permission from the license holder to reproduce the material. To view a copy of this license, visit http://creativecommons.org/licenses/ by-nc-nd/3.0/ 